\title{
Nevus changed by recurrent Herpes simplex infection: Reflectance confocal microscopy
}

\author{
Marco Palla, Fabrizio Ayala*, Gerardo Botti, Paolo Antonio Ascierto
}

Department of Melanoma, National Cancer Institute “G. Pascale” Foundation, Naples, Italy;

*Corresponding Author: fabrizio.ayala@gmail.com

Received 6 November 2013; revised 5 December 2013; accepted 2 January 2014

Copyright (C) 2014 Marco Palla et al. This is an open access article distributed under the Creative Commons Attribution License, which permits unrestricted use, distribution, and reproduction in any medium, provided the original work is properly cited. In accordance of the Creative Commons Attribution License all Copyrights (C) 2014 are reserved for SCIRP and the owner of the intellectual property Marco Palla et al. All Copyright (C) 2014 are guarded by law and by SCIRP as a guardian.

\section{ABSTRACT}

During a screening program, a 24-year-old woman presented an itchy nodule above the upper lip, $5 \times 5 \mathrm{~mm}$ in size with irregular pigmentation, which had enlarged over the previous three weeks. Personal clinical history revealed a recurrent Herpes virus infection. Dermoscopy showed light brown irregular pigmentation, milia-like cysts and an atypical vascular pattern. Reflectance confocal microscopy revealed architectural, cytologic and vascular features suggestive of melanoma. A Tzanck test revealed multinucleated keratinocytes with the typical nuclei infected by Herpes virus. Our case shows that dermoscopy alone, despite its sensitivity and specificity, may not provide adequate diagnostic accuracy on which to base a surgical decision. Epiluminescence criteria should be considered together with a clinical evaluation of the lesion and the patient's clinical history. Reflectance-mode confocal microscopy confirmed the difficulty of presurgical diagnosis in this case, which highlights the importance of traditional cytological analysis in order to avoid unnecessary surgical treatment.

\section{KEYWORDS}

\section{Confocal Microscopy; Melanoma; Naevi; Herpes Simplex Virus}

\section{INTRODUCTION}

Nevi are the most common simulators of melanoma. Occasionally, their clinical and dermoscopic features are not sufficient to confirm a diagnosis. We report a nevus on the upper lip altered by Herpes simplex virus infection, with the clinical appearance of an abnormal pink nodule in which a periferic area of pigmentation was evident. We describe dermoscopic, reflectance-mode confocal microscopic, and cytological diagnostic features of this skin lesion. The aim of this study is to highlight the importance of traditional cytological analysis in order to avoid unnecessary surgical treatment, and to describe the specific and particular reflectance confocal microscopic images of keratinocytes infected by Herpes simplex virus.

\section{CASE}

We report the case of a 24-year-old woman presented with an itchy dome-shaped nodule above the upper lip, with light brown irregular pigmentation; clinical history revealed recent enlargement of the lesion (Figure 1). Dermoscopic examination (Molemax II, Derma Instruments, Vienna, Austria) of the lesion showed a periferic light brown irregular pigmentation, milia-like cysts and an atypical vascular pattern. In vivo reflectance confocal microscopy (RCM) (Vivascope 1500; Lucid Inc., Rochester, NY, USA) was performed at different depth levels to a maximum of $150 \mu \mathrm{m}[1,2]$; of concern was the observation in the epidermal layer of a diffuse pattern of single and multinucleated large keratinocytes, demarcated with sharp contours, containing bright grainy cytoplasm and round dark nuclei with bright dots close to the nuclear envelope in a ring pattern (Figure 2(a)). At the dermo-epidermal junction, cords of variable refractile cells arranged parallel to each other were observed, as were keratinocytes with thickened contours, different in size and refractivity and arranged with an atypical honeycomb pattern. The slight architectural disarray also showed bright nucleated cells with dendritic-like branches, and diffuse small bright cells and particles. A 


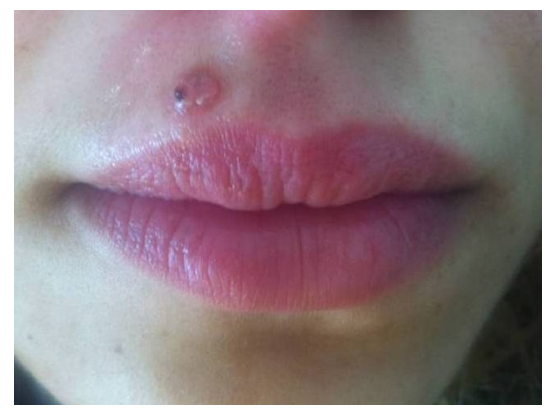

Figure 1. Atypical pigmented nodule of $5 \times 5 \mathrm{~mm}$ on upper lip.
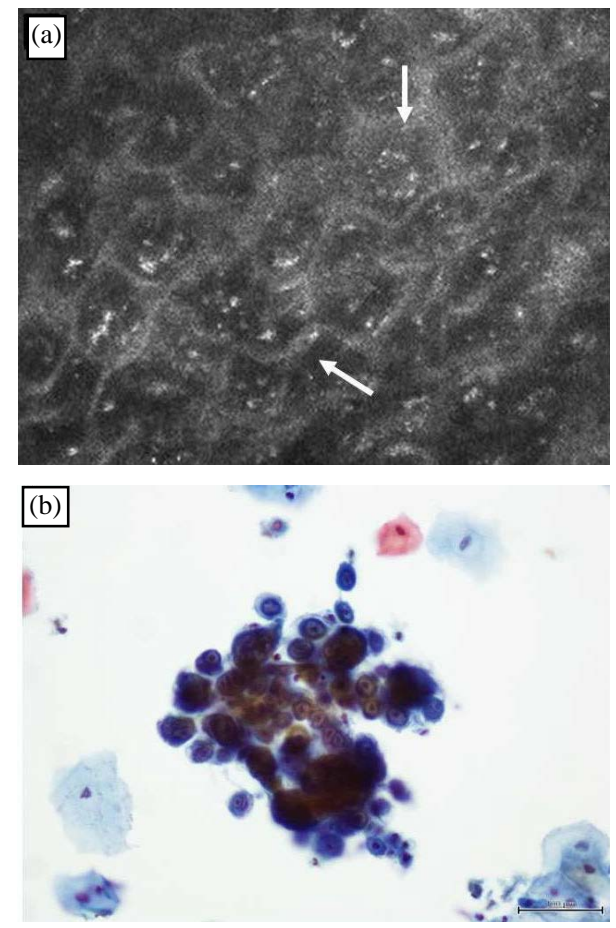

Figure 2. (a) Multinucleated large polygonal keratinocytes with demarcated bright contour $(\rightarrow)$ containing a round dark nucleus with a peripheral bright dot arranged with a ring pattern at the upper level of epidermis; (b) Tzanck smear sample with degeneration of keratinocyte nuclei, and the multinucleated keratinocytes with the typical "ground glass" nuclei due to intranuclear viral particles that cause a peripheral distribution of the chromatin close to nuclear envelope. Polygonal keratinocytes detected at periphery of image. (Thin-prep Papanicolau, 40×).

proliferation of melanocytes, bulging within the dermal papilla, were organized as solitary units and oval demarcated nests [3]. Because the patient reported a recurrent infection of Herpes simplex virus on her upper lip, we allowed five days of follow-up, instead of a surgical approach. On the fifth day, a pustule was present on top of the nodule and a Tzanck test was performed; it showed keratinocytes with degeneration of nuclei, in which the multinucleated keratinocytes had the typical molded nuclei of Herpes infection (pomegranate seed appearance) (Figure 2(b)).

We treated the lesion with acyclovir for two weeks, and after this time it had decreased in diameter, no itching or pain was reported, and no further viral atypia were detected by either dermoscopy or RCM.

\section{CONCLUSIONS}

Melanocytic nevi are clinically significant in that their appearance can be similar to that of melanoma. Although clinical and dermoscopic features are usually sufficient to confirm a diagnosis of melanocytic nevi or melanoma, this may not always be the case. For instance, diagnosis can be complicated in cases where inflammatory disease or infection, such as Herpes virus, results in enlargement of the nevus, which can be confused with a hypopigmented nodular melanoma or hypopigmented nodular basal cell carcinoma [4,5].

On RCM, the architectural disarray at the dermal-epidermal junction, the diffuse dendritic and small bright cells corresponded to inflammation, and the large irregular blood vessels were not suggestive of either melanocytic or keratinocytic malignancy [2,3]. However, the presence of multinucleated keratinocytes with high refractile dots, distributed at the periphery close to the nuclear envelope, potentially suggested a viral infection.

Our original and first case in literature about RCM in Herpes simplex infection shows that clinical evaluation and dermoscopy may not be sufficient to obtain a satisfactory diagnosis, while RCM is actually a very advanced in vivo diagnostic tool; also, cytological analysis is still a traditional and very useful examination in doubtful cases.

\section{REFERENCES}

[1] Pellacani, G., Guitera, P., Longo, C., et al. (2007) The impact of in vivo reflectance confocal microscopy for the diagnostic accuracy of melanoma and equivocal melanocytic lesions. Journal of Investigative Dermatology, 127, 2759-2765.

[2] Pellacani, G., Longo, C., Malvehy, J., et al. (2008) In vivo confocal microscopic and histopathologic correlations of dermoscopic features in 202 melanocytic lesions. Archives of Dermatology, 144, 1597-1608. http://dx.doi.org/10.1001/archderm.144.12.1597

[3] Segura, S., Pellacani, G., Puig, S., et al. (2008) In vivo microscopic features of nodular melanomas: Dermoscopy, confocal microscopy, and histopathologic correlates. Archives of Dermatology, 144, 1311-1320. http://dx.doi.org/10.1001/archderm.144.10.1311

[4] Menzies, S.W., Kreusch, J., Byth, K., et al. (2008) Dermoscopic evaluation of amelanotic and hypomelanotic 
melanoma. Archives of Dermatology, 9, 1120-1127.

[5] Ascierto, P.A., Palmieri, G., Botti, G., et al. (2003) Early diagnosis of malignant melanoma: Proposal of a working formulation for the management of cutaneous pigmented lesions from the Melanoma Cooperative Group. International Journal of Oncology, 22, 1209-1215. 